\title{
Power Allocation Algorithm based on Nth order Fibonacci Polynomial for Multi-User NOMA
}

This paper was downloaded from TechRxiv (https://www.techrxiv.org).

\section{LICENSE}

CC BY 4.0

SUBMISSION DATE / POSTED DATE

$23-12-2020 / 12-01-2021$

\section{CITATION}

Mumtaz, Zeeshan; Zakwan, Muhammad; Adnan, Muhammad; Fazil, Adnan (2020): Power Allocation Algorithm based on Nth order Fibonacci Polynomial for Multi-User NOMA. TechRxiv. Preprint. https://doi.org/10.36227/techrxiv.13482330.v2

DOI

10.36227/techrxiv.13482330.v2 


\title{
Power Allocation Algorithm based on $N^{\text {th }}$ order Fibonacci Polynomial for Multi-User NOMA
}

\author{
Muhammad Zeeshan Mumtaz (D), Muhammad Zakwan ${ }^{\dagger}$ (D), Muhammad Adnan ${ }^{\ddagger}$ and Adnan Fazil ${ }^{\S}$ (iD) \\ Institute of Avionics and Aeronautics, \\ Air University, Islamabad \\ Email: *zeeshan2708@ hotmail.com, ${ }^{\dagger}$ mzakwan@mail.au.edu.pk, ${ }^{\ddagger}$ madnan@mail.au.edu.pk, \\ §adnan630@hotmail.com
}

\begin{abstract}
In this paper, we propose a novel empirical power allocation algorithm based on individual term normalization of $N^{\text {th }}$ order Fibonacci polynomial for multi user Non Orthogonal Multiple Access (NOMA), providing a characteristic solution to allocation problem for $n$-users. A deterministic mathematical expression for same predetermined power allocation scheme for both superposition coding (SC) at base station domain and successive interference cancellation (SIC) at user domain has been formulated. Fibonacci polynomial has been defined and linked to power allocation algorithm corresponding to radial distance of users from BS with sole requirement of second order statistics (SOS). Holistic analytical reasoning has been carried out for generic NOMA system and an exact closed-form expression for bit error rate (BER) has been derived using probabilistic models, which has been applied to proposed allocation algorithm. Detailed analysis validates that proposed power allocation algorithm is independent of user channel state information (CSI) which averts cumbersome computations and no prior information regarding allocation is required to be relayed to user domain for successful SIC. Numerical and simulation results (upto 7 user NOMA) have been provided to demonstrate superior performance in terms of reliability and latency of the proposed algorithm in comparison to channel inversion, a CSI based algorithm.
\end{abstract}

Index Terms-Non Orthogonal Multiple Access (NOMA), $N^{\text {th }}$ order Fibonacci polynomial.

\section{INTRODUCTION}

With the era of $5^{\text {th }}$ generation of wireless communication networks $(5 \mathrm{G})$ ushering into the world, new technological avenues are being ventured upon. Professional norms like ultimate real-time interconnectivity, low latency and reliable communication have been embraced as new normal by common man. The realization of these tech-vernaculars in form of smartphones and self driving cars to low cost sensors for industry and agriculture has imposed stringent performance constraints on communication systems [1][2]. As time-frequency resource is becoming a prized commodity, other domains are being exploited to multiplex multiple user simultaneously. Non orthogonal multiple access (NOMA) is being perceived as an attractive choice for multiplexing in power domain. In addendum to this, its architectural flexibility allows its combination with other technologies such as massive multiple input multiple output (MIMO) which provides diversity in space domain [3][4][5]. Owing to the superior performance of NOMA over conventional orthogonal multiple access schemes
(OMA), it was included in the study item list of Release 15 of 3rd generation partnership project (3GPP) [6].

Power domain NOMA has proved its capability of high spectral efficiency over OMA schemes while maintaining the reliability at utmost thresholds. For the purpose of parallel transmission to multiple users simultaneously, it utilizes superposition coding (SC) at transmitter to multiplex several data streams on single frequency channel by manipulating power domain and successive interference cancellation (SIC) at receiver for flawless extraction of concerned data stream [7]. Power allocation algorithm is the core concept in both SC and SIC blocks of NOMA communication systems as power domain multiplexing assigns relative power coefficients for each user from constant transmitter power, while decoding removes interference signal by remodulation, multiplication with power coefficient and subtraction from complete received signal. Therefore, a judicious power allocation is vital for efficient and reliable NOMA communication.

\section{A. Literature Review}

Even though numerous strategies have been proposed for rational power allocation to multiple users since NOMA has emerged as multiplexing method, the demand for a minimalistic allocation approach is even more pertinent considering modern communication requirements. Most of the research work focuses on channel state information (CSI) in determination of power allocation algorithm which burdens the computational resources in time constrained scenario, especially in fast fading channel cases. For instance, channel inversion has been used to serve the purpose by inverting fading channel coefficients which accounts rapidly changing channel conditions with each time slot [8]. Difference in channel estimation at transmitter and receiver results in different sets of power coefficients, leading to inaccuracies in decoding process. In [8], another strategy has also been proposed, predefined quality-of-service (QoS), which divides users into priority based classes. However, the expression for power allocation coefficients also involves fading channel coefficients and SINR values, resulting in exhaustive computations. Two CSI based strategies, named as imperfect CSI and SOS CSI, have been discussed in [9], which considers the truncated CSI model with lesser number of parameters, with assumption 
of small-scale Rayleigh fading. Yet both strategies require fading channel coefficients in power allocation which lead to similar issues as discussed earlier. In [10], the authors have implemented 2 user and 3 user NOMA with fixed allocation coefficients and presented satisfactory BER results. However, this approach lacks any empirical reasoning behind the choice of coefficient set and fail to provide a generalized solution for $n$-users scenario. In [11], ergodic sum rate is used as objective function for non-convex optimization for power allocation and sub optimal solution is derived. Again, this work involves tiresome processing for fast fading channels needed for ergodic sum rate calculations and makes it impractical in extreme realtime situations. The authors in [12] have used sum capacity as objective function and maximized it for fairness constraint, but the sum capacity is proportional to fast fading channel coefficients, varying in each time slot causing variable set of power coefficients. Recently, artificial intelligence (AI) has been used in pursuit of ideal solution to power allocation problem [13] [14]. In [13], a fully connected deep neural network (DNN) has been trained for estimation of power allocation scheme using interior point method (IPM) with loss function related to sum rate. Nonetheless, it requires extensive data set by simulation of channel coefficients for training complex DNN structure. In addition to this, training timing required has order of seconds which make it unsuitable for rapid changing environment.

\section{B. Motivation and Contributions}

The foundation of power domain NOMA lies in power allocation algorithm which assigns relative power to each user based on principle of power equity/ fairness. The existing efforts for addressing this issue can be classified into two categories. Firstly, in fixed power allocation, coefficients are assigned to 2 or 3 user scenarios to altogether avoid the complex allocation procedure, as performed in [10]. This method is practically impossible to be generalized for increasing number of users, due to absence of any closed form expression. Secondly, in CSI based power allocation, used by the majority of the NOMA communication systems, fair power division among multiple users has been achieved by using channel coefficients in different patterns. However, fast fading channels vary channel coefficients for each time slot, which forces re-evaluation of power coefficients instantaneously. As the user base expands, the optimization of CSI based objective function becomes intractable. These shortcomings of existing algorithms motivates to develop a generalized power allocation which can accommodate increasing number of users.

The notable contributions of this dissertation are summarized as below.

- A generalized mathematical expression has been formulated for power coefficients for $n$-users, based on normalization of individual term of a defined Fibonacci polynomial which depends exclusively on number of users and second order statistics (SOS) which only arranges the users in order of their radial distance from the base station. No CSI parameters is involved in this process, which makes the algorithm deterministic in nature. Both transmitter and receiver have inherently same set of power allocation coefficients thoroughout the complete communication session. Power allocation algorithm can be utilized readily for all variants of NOMA communication system supporting any number of users as it does not involves re-evaluation process as in case of CSI based methods.

- Bit error rate (BER) has been investigated for $n$-user general NOMA communication system with probabilistic models of Rayleigh fading channels and AWGN noise channel. An exact closed form expression has been derived for BER applicable for any NOMA system which has been further used for proposed Fibonacci polynomial power allocation algorithm.

- A comprehensive 7-user NOMA with proposed power allocation algorithm has been simulated with superposition coding (SC) and successive interference cancellation (SIC) successfully. Detailed BER analysis has been carried out for individual users and overall simulated system. Constellation diagrams for SC signal and equalized signal at each iteration of SIC have been visualized which provide perception of inphase and quadrature phase components (I/Q channels) for power domain multiplexing. Both numerical and simulation results demonstrate the superior performance of proposed algorithm in comparison to the CSI based algorithm.

Notations: $\mathbb{C}$ is used to denote complex random variable while $\mathcal{N}$ represents normal distributed random variable. $\beth$ is used for new defined Fibonacci polynomial, $\mathbb{H}$ for channel matrix and $\eta$ for path loss exponent.

\section{NOMA SYSTEM MODEL}

We consider a situation in which $n$ number of users have to be provided concurrent services by a single base station. For this document, we are only focusing on a single sub group of users out of whole cluster of consumers which will be multiplexed over single frequency channel as depicted in Fig. 1 However, the proposed system can easily be generalized for several subgroups by using multiple frequency bands. The users of the subgroup under consideration are well scattered in the sector of communication in both planar polar coordinates $(r, \theta)$ as shown for 7 users in Fig. 2

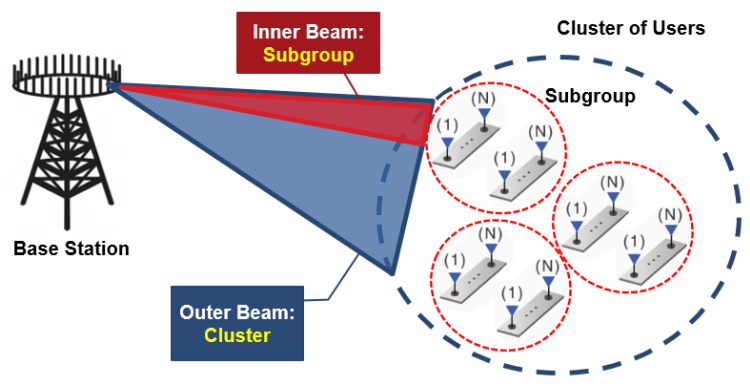

Fig. 1. NOMA User Segregation.

The proposed system uses the presumption of Second Order Statistics (SOS), which is used to get the ascending order 
of users corresponding to their radial distance from the base station. SOS is primarily based on the manipulation of the two smallest values of a random sample [15] [16]. The system can use two approaches for sampling required for user distance order determination for SOS application:

- Power measurement of duplex signal by both BS and user dominions.

- Single time calculation of fading channel coefficients at initial phase with assumption that slow user order will not be altered in considerable time interval.

The arranged order of user can be represented as $d_{1}>$ $d_{2}>\cdots>d_{k} \cdots>d_{n}$ which relates the lowest valued prefix to farthest user from the base station while highest prefix to nearest user based on the SOS approximations.

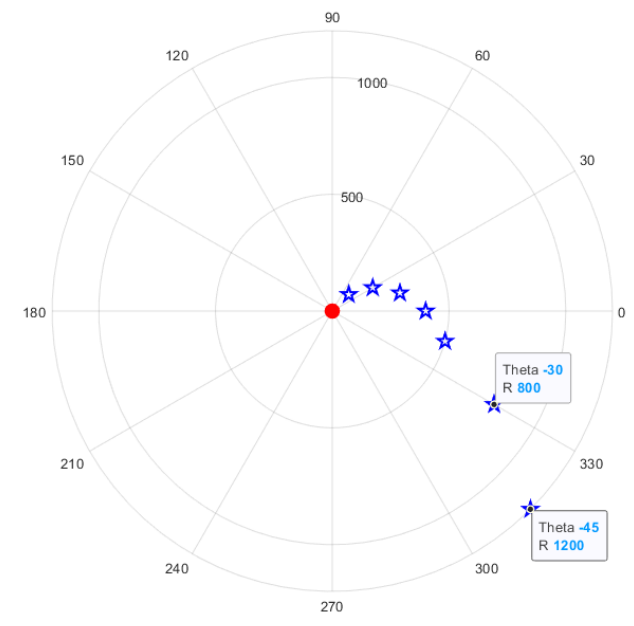

Fig. 2. Location of 7 users with base station at origin.

The transmitter side of proposed NOMA system possesses Bernoulli data generator which spawns individual binary data sets for each user. This data is subjected to QPSK modulation, which provides inphase and quadrature components (I/Q) of the data. The modulated signal is subjected to multiplexing in power domain by using superposition coding which is discussed in subsequent section.

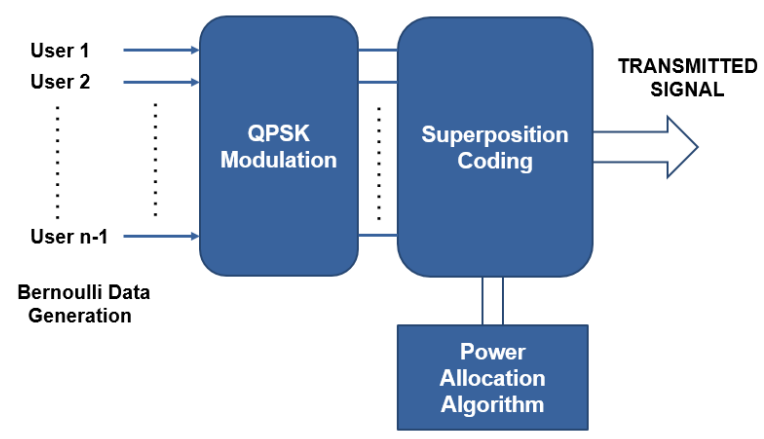

Fig. 3. Functional diagram of NOMA transmitter

\section{A. Fibonacci Power Allocation Algorithm}

Power allocation algorithm is the vital block of superposition coding as it judiciously combine the modulated signals into single stream of data for single beam transmission to a subgroup of users.

$$
S=\sum_{k=1}^{n} \sqrt{\alpha_{k} P_{t}} \times s_{\text {mod }, k}
$$

$S$ represents the super-positioned signal, $P_{t}$ denotes the transmission power, $s_{\bmod , k}$ is the QPSK modulated signal for $k^{t h}$ user, while $\alpha_{k}$ is the power coefficient allocated to the $k^{t h}$ user.

Various schemes as discussed in preceding sections have been devised to assign this coefficient to achieve fairness of power division among all users which conforms to $\alpha_{1}>\alpha_{2}>$ $\cdots \alpha_{k}>\cdots \alpha_{n}$, reserving larger power share for farthest users in comparison to nearer ones. The necessary condition for these coefficients is that their summation must be utmost unity as the transmission power is conserved such as $\sum_{k=1}^{n} \alpha_{k}=1$ [9].

Fibonacci sequence is a renowned mathematical series of number which has found its use in numerous natural phenomena. We have utilized its efficacy for our problem of power assignment. The mathematical realization of this series is that each member is sum of previous two members.

$x_{n}=x_{n-1}+x_{n-2}$ where $x$ is a member of Fibonacci series while $x_{n}=0,1,1,2,3,5,8,13,21, \cdots$ for $n=0,1,2,3, \cdots$

Now we define a $N^{\text {th }}$ order polynomial for $n$ terms (number of users in subgroup) such as:

$$
\begin{aligned}
\beth_{n}^{N} & =x_{n+1}^{N}+x_{n}^{N}+x_{n-1}^{N}+x_{k+1}^{N}+\cdots+x_{n-(n-2)}^{N} \\
& =\sum_{k=1}^{n} x_{k+1}^{N}
\end{aligned}
$$

Consider the normalization of individual terms of this polynomial to be power allocation coefficients reciprocating their order to justify power assignment fairness.

$$
\begin{gathered}
\alpha_{k}=\frac{x_{n-(k-2)}^{N}}{x_{n+1}^{N}+x_{n}^{N}+x_{n-1}^{N}+x_{i+1}^{N}+\cdots+x_{n-(n-2)}^{N}} \\
\text { or } \quad \alpha_{k}=\frac{x_{n-(k-2)}^{N}}{\beth_{n}^{N}}
\end{gathered}
$$

It can be observed that this expression is explicitly empirical in nature and does not depends upon fading channel coefficients or other CSI parameters. This algorithm only has to account for the number of users in a subgroup for Fibonacci series term indexing to evaluate power coefficients for all users instantaneously. This equation intrinsically satisfies the necessary condition for power allocation as summation of coefficients converge to unity as $\sum_{k=1}^{n} \alpha_{k}=1$. This simplistic expression can be used to generate sets of power coefficients $\alpha$ for any $n$ number of user without knowledge of any other system parameter for a fixed arbitrary order value $N$. For instance, for $N=4$, we get a table for coefficients corresponding to number of users as shown in Table I] 
TABLE I

\begin{tabular}{|c|l|}
\hline \multicolumn{2}{|c|}{$\mathbf{4}^{\text {th }}$ Order Fibonacci Polynomial Algorithm } \\
\hline $\begin{array}{c}\text { No. of } \\
\text { users } \boldsymbol{n}\end{array}$ & Power Allocation Coefficients $\boldsymbol{\alpha}$ \\
\hline 1 & 1 \\
\hline 2 & $0.9412,0.0588$ \\
\hline 3 & $0.8265,0.1633,0.0102$ \\
\hline 4 & $0.8645,0.1120,0.0221,0.0014$ \\
\hline 5 & $0.8500,0.1297,0.0168,0.0033,0.0002$ \\
\hline 6 & $0.8556,0.1227,0.0187,0.0024,0.0005$, \\
& $3 \times 10^{-5}$ \\
\hline 7 & $0.8535,0.1253,0.0179,0.0027,0.0004$, \\
& $7.02 \times 10^{-5}, 4.39 \times 10^{-6}$ \\
\hline
\end{tabular}

\section{B. Channel and Noise Model}

Channel matrix $\mathbb{H}$ is a manifestation of prior probability of correct reception of each possible symbol over a communication channel. This concept is used for representing the distortion impact on the original signal, like fading, multipath effect and interference, posed by a real communication channel. The overall matrix itself is concatenation of individual fading channel vectors $h_{k}$ for each user as .

$$
\begin{gathered}
\mathbb{H}=\left[\begin{array}{lccc}
h_{1}^{T} & h_{2}^{T} \cdots & h_{k}^{T} \cdots h_{n}^{T}
\end{array}\right] \quad \epsilon \quad \mathbb{C}^{N_{s} \times n} \\
\mathbb{H}=\left[\begin{array}{cccc}
h_{11} & h_{12} \cdots & h_{1 k} \cdots & h_{1 n} \\
\vdots & \vdots & \vdots & \vdots \\
h_{N_{s} 1} & h_{N_{s} 2} & \cdots & h_{N_{s} k} \\
\cdots & h_{N_{s} n}
\end{array}\right]
\end{gathered}
$$

where $N_{s}$ is total symbols/user, $n$ is number of users in subgroup.

We have considered Rayleigh fading channel which provide good approximation for modelling real channel effects. Generally, Rayleigh channel is represented by variation of exponential distributions. However, for multiple time instant use of the channel forms a sequence of independent exponential random variables (RV). Here we use central limit theorem for large sequence of RVs converge to normal distribution. The channel coefficients of a sequence of independent Rayleigh channel uses can be represented by following complex vector expression [17].

$$
h_{k}=\sqrt{d_{k}^{-\eta}}\left(\frac{X_{h}+i Y_{h}}{\sqrt{2}}\right) \in \mathbb{C}^{N_{s} \times 1}
$$

where $d_{k}$ is the radial distance of user $k$ from base station, $\eta$ is path loss exponent and $X_{h}$ and $Y_{h}$ are two normally distributed random variables such as $X_{h}, Y_{h} \sim \mathcal{N}(0,1)$.

Additive White Gaussian Noise (AWGN) model has been used for simulation of random noise present in the communication channel. The AWGN is also represented by using two normal random variable again using central limit theorem as following [18].

$$
n_{k}=\sqrt{n_{0}}\left(\frac{X_{n}+i Y_{n}}{\sqrt{2}}\right) \in \mathbb{C}^{N_{s} \times 1}
$$

where $n_{0}=k T B$ which represents the thermal noise and $X_{n}$ and $Y_{n}$ are two normally distributed random variables such as $X_{n}, Y_{n} \sim \mathcal{N}(0,1)$. The overall noise matrix for NOMA system can be formed by concatenation of noise coefficients for each symbol designated for each user.

$$
\begin{aligned}
& \mathbb{N}=\left[\begin{array}{ll}
n_{1}^{T} & n_{2}^{T} \cdots n_{k}^{T} \cdots n_{n}^{T}
\end{array}\right] \epsilon \quad \mathbb{C}^{N_{s} \times n} \\
& \mathbb{N}=\left[\begin{array}{cccc}
n_{11} & n_{12} \cdots & n_{1 k} \cdots & n_{1 n} \\
\vdots & \vdots & \vdots & \vdots \\
h_{N_{s} 1} & h_{N_{s} 2} \cdots & n_{N_{s} k} \cdots & n_{N_{s} n}
\end{array}\right]
\end{aligned}
$$

\section{Signal Reception}

Now we will focus on the user domain of NOMA system and develop an relationship for received superimposed data at each user $k$ which can be formulated with the transmitted data from base station subjected to Rayleigh channel distortions and random AWGN noise [10][19].

$$
\mathbf{y}=\mathbb{H} S+\mathbb{N} \quad \epsilon \quad \mathbb{C}^{N_{s} \times n}
$$

After matrix multiplication, this equation for individual user $k$ reduces to a vector as below.

$$
\boldsymbol{y}_{\boldsymbol{k}}=\boldsymbol{h}_{\boldsymbol{k}} \times S+\boldsymbol{n}_{\boldsymbol{k}} \quad \epsilon \quad \mathbb{C}^{N_{s} \times 1}
$$

For negotiating the channel distortion effects, this received signal is subjected to perfect zero-forcing equalization as we have absolute knowledge of fading channel coefficients in simulation dominion. This type of equalization involves application of inverse of frequency response of channel matrix [20][21]. The Fourier transform of the equalized signal is:

$$
\begin{array}{ccc}
\tilde{\boldsymbol{Y}}=\boldsymbol{Y} \overline{\mathbb{H}}^{-1} \quad \Rightarrow & \text { Frequency Domain } \\
\tilde{\boldsymbol{y}}=\boldsymbol{y} \overline{\boldsymbol{H}}^{\dagger} \quad \Rightarrow \quad \text { Time Domain }
\end{array}
$$

$\bar{H}^{\dagger}$ depicts the pseudo inverse of channel coefficients which is applied to the received single to realize the perfect zeroforcing equalization. The equalized signal $\tilde{y}$ is the distorted version of the superposition coded signal which has multiplexed data for all users of the subgroup. However, for deciphering the concerned signal for user $k$, the user has to remove the signal intended for users with stronger signal power users (the farthest users). The signal of these users (1 to $k-1$ ) appears as interference for $k^{t h}$ user. We rewrite the equation of received signal as:

$$
y_{k}=\sqrt{\alpha_{k} P_{t}} \times s_{m o d, k}+\sum_{j=1}^{k-1} \sqrt{\alpha_{k} P_{t}} s_{m o d, j}+n_{k} \bar{h}^{\dagger}
$$

Successive Interference Cancellation (SIC) is used to discard the stronger signals of farther users by following an iterative scheme for removing interference signals sequentially. This block demodulates the equalized signal and assesses whether the signal recovered is intended for user $k$. If it is the desired message, it stops its iterative loop, else it remodulates, assigns corresponding power allocation coefficient and subtracts it from the equalized signal. This loop is repeated till the time user $k$ identifies the signal intended for it. The working flow diagram of SIC algorithm is shown in Fig. 4. 


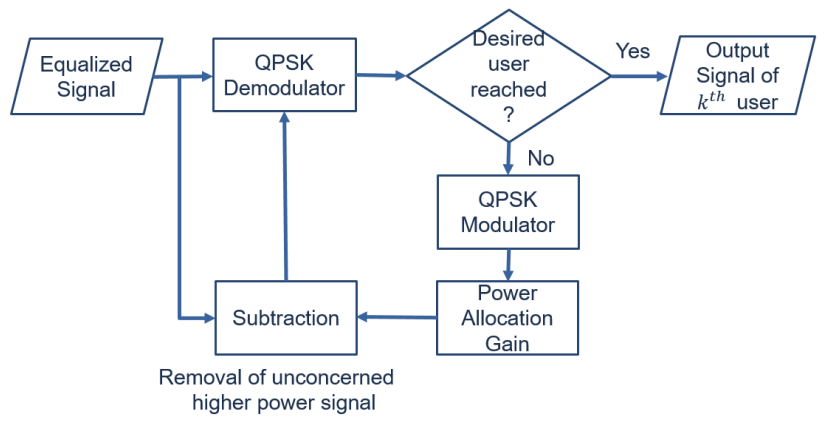

Fig. 4. Flow chart of SIC algorithm

At this stage, we can appreciate the deterministic nature of Fibonacci polynomial power allocation algorithm as the same set of coefficients, used in superposition coding at transmitter, should be applied for SIC iterative scheme to recover the desired signal. Difference in channel estimation at base station and users can lead to ineffective optimization of CSI based power allocation algorithms, which will cause drastic reduction in communication reliability.

\section{Performance Analysis}

In this section, we investigate the performance of the proposed NOMA communication system with Fibonacci polynomial power allocation. First, the bit error rate (BER) results for three scenarios $(3,5$ and 7 users per subgroup) of this simulated NOMA system are deliberated upon. Visualization of constellation diagrams of superposition coded signal and equalized signal at user domain at each iteration of SIC is also discussed which provide comprehension of I/Q components for power domain multiplexing. Secondly, an exact closed form expression for BER for a generic NOMA system is derived using the probabilistic models of Rayleigh fading channel and AWGN noise channel. This generalized BER analysis is utilized to get insight of the proposed allocation algorithm. Finally, channel inversion method, a CSI based power allocation algorithm proposed in [8] is applied to the same NOMA system and its performance is compared with Fibonacci polynomial allocation algorithm for apprehension of efficacy of our proposed design.

\section{A. Bit Error Rate Results for Fibonacci Power Algorithm}

For better intuition of our proposed algorithm, three scenarios of multiple number of users per subgroup (3, 5 and 7$)$ have been considered for NOMA system. The specifications of proposed NOMA system are tabulated in Table II

Considering these specifications and employing Fibonacci power allocation coefficients as mentioned in Table I bit error rate (BER) analysis for variable transmission power $\left(P_{t}\right)$ has been carried out for 3 users in a sub group and presented in Fig. 5

In-depth preview of the Fig. 5 points out that actual BER values for all users drop to the order of $10^{-7}$ as the NOMA base station transmission power reaches for $40 \mathrm{dBm}$ (10 watts). The break in the graph shows that BER values for strongest
TABLE II

\begin{tabular}{|c|c|}
\hline \multicolumn{2}{|c|}{ NOMA System Specifications } \\
\hline System test data size & 45 MBits/ session \\
\hline Order of polynomial & 4 \\
\hline System Bandwidth & $1 \mathrm{MHz}$ \\
\hline Thermal Noise $n_{0}$ & $-114 \mathrm{dBm}$ \\
\hline Path Loss Exponent $\eta$ & 3 \\
\hline Azimuth Sector $\theta$ & $-45^{\circ}$ to $+45^{\circ}$ \\
\hline Simulation Processor & $\begin{array}{c}\text { Intel i7-8550U clocked } 3.00 \mathrm{GHz} \\
\text { (8 GB RAM) }\end{array}$ \\
\hline
\end{tabular}

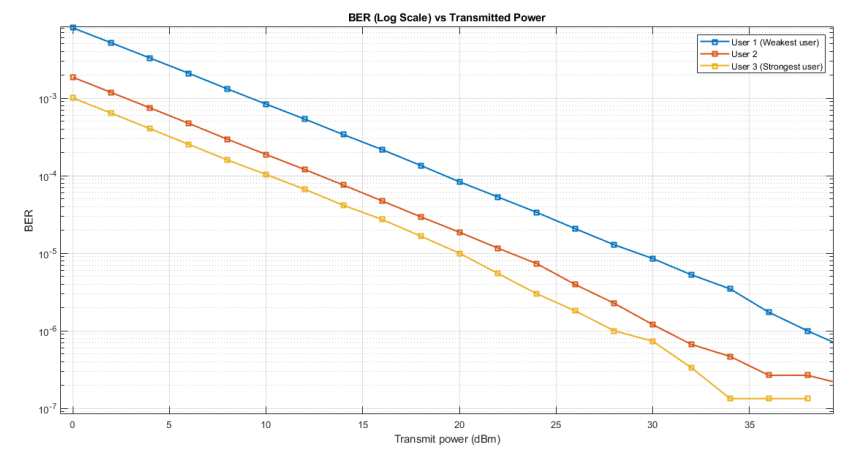

Fig. 5. BER versus transmission power $(\mathrm{dBm})$ for 3 users/ subgroup with radial distances in meters $d_{1}=70 \mathrm{~m}, d_{2}=40 \mathrm{~m}, d_{3}=20 \mathrm{~m}$ and test data size $=15$ MBits/ user. Simulation time $-121 \mathrm{sec}$

user have practically reduced to zero for finite test data. The total simulation time for SC transmission from base station and successful recovery of 15 MBits by all 3 users is 121 seconds over complete transmission power range $(1 \mathrm{~mW}$ to $10 \mathrm{~W})$.

Now we consider the scenario for 5 user per subgroup using the same specifications with test data size of 9 MBits/user, maintaining the overall system test data set at 45 MBits. The proposed Fibonacci power allocation algorithm assigns coefficients to 5 users scattered at different locations. The bit error rate values for all users monotonically reduce with transmission power increase as shown in Fig. 6.

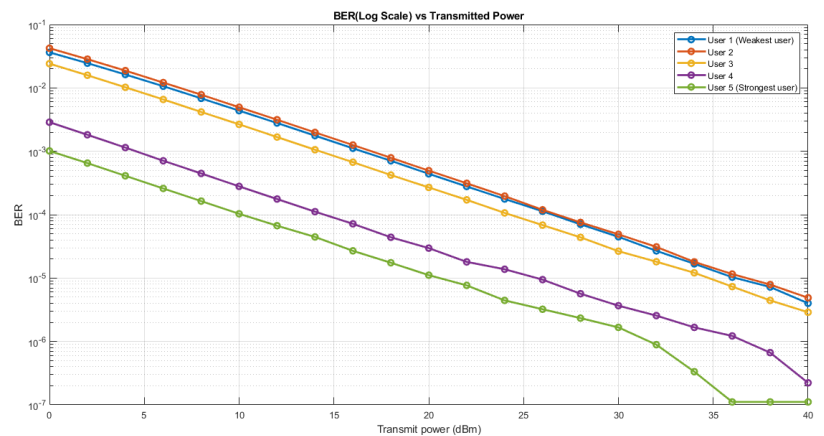

Fig. 6. BER versus transmission power $(\mathrm{dBm})$ for 5 users/ subgroup with radial distances in meters $d_{1}=100 \mathrm{~m}, d_{2}=70 \mathrm{~m}, d_{3}=40 \mathrm{~m}, d_{4}=$ $20 \mathrm{~m}, d_{5}=10 \mathrm{~m}$ and test data size $=9$ MBits/ user. Simulation time- 161 $\sec$

It can be acknowledged by the analysis of Fig. 5 and Fig. 6 that BER values for 5 user case have increased from 3 user case because the same transmission power is being divided into more number of user which results in lesser energy per 
symbol and lower $E_{b} / N_{0}$ for individual users, leading to more erroneous bits. The simulation time for 5 users/ subgroup (161 seconds) has also increased as 2 more users have to complete SIC procedure.

For more sturdy testing on performance of the proposed Fibonacci algorithm, 7 users per subgroup is considered for simulated NOMA system. The overall test data of 45 MBits is divided into 7 streams of 6.4 MBits. Actual BER values for 7 user case further testify the general applicability of deterministic Fibonacci polynomial allocation scheme within relatively lower simulation time (209 seconds) as shown in Fig. 7

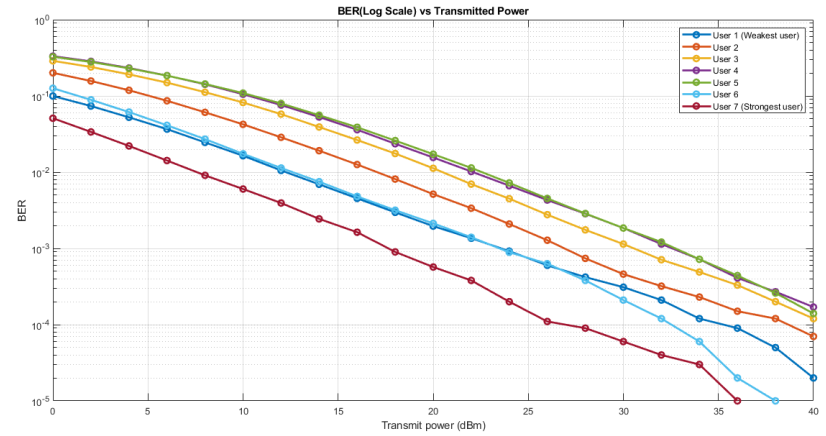

Fig. 7. BER versus transmission power $(\mathrm{dBm})$ for 7 users/ subgroup with radial distances in meters $d_{1}=120 \mathrm{~m}, d_{2}=100 \mathrm{~m}, d_{3}=80 \mathrm{~m}, d_{4}=$ $60 \mathrm{~m}, d_{5}=40 \mathrm{~m}, d_{6}=20 \mathrm{~m}, d_{7}=10 \mathrm{~m}$, and test data size $=6.4$ MBits $/$ user. Simulated time - $209 \mathrm{sec}$

\section{B. Constellation Diagrams of Superposition Code and SIC Iterations}

Constellation diagrams provide profound insight of modulation technique as it defines the size of alphabet of symbols used to encode each sample and also determines the number of bits required for single sample [22]. In [25], the authors have used constellation diagrams for BER performance analysis for 2 users and 3 users NOMA systems with Nakagami $m$-fading channels. We have utilized the analysis of above cited research and generalized the equations for symbol alphabet size and bits required for each sample for $n$ users. Furthermore, for 5 and 7 user scenarios, we have compared the constellation diagrams of superposition code and successive iterations of SIC to visualize the distortion effects.

Considering QPSK modulation, each sample is represented by 4 symbols ( 2 bits) with constant amplitude and four points in complex plane of inphase and quadrature components. In superposition coding for $n$-users, it has to multiplex $n$ QPSK symbols and forms $\left(2^{n}\right)^{2}=2^{2 n}$ constellation points. As each user symbol requires 2 bits, NOMA superposition signal requires $2 n$ bits/sample.

$$
\text { Sample size }=2 n \text { bits/sample }
$$

Total constellation points $=2^{2 n}$ symbols

According to equation (18), constellation diagrams for superposition coding for 5 user and 7 user cases (Fig. 8 and
Fig. 9p have 1024 and 16384 constellation points which have 256 and 4096 copies for clearly demarcated of individual 4 symbol set of QPSK modulated signal of each user sample. In Fig. 9, each blue square represents 64 constellations points which are closely spaced in amplitude and phase components. The reference QPSK 4 symbol set has been embedded in the center of constellation diagram for better comprehension of symbol amplitude and phase variations over complex plane.

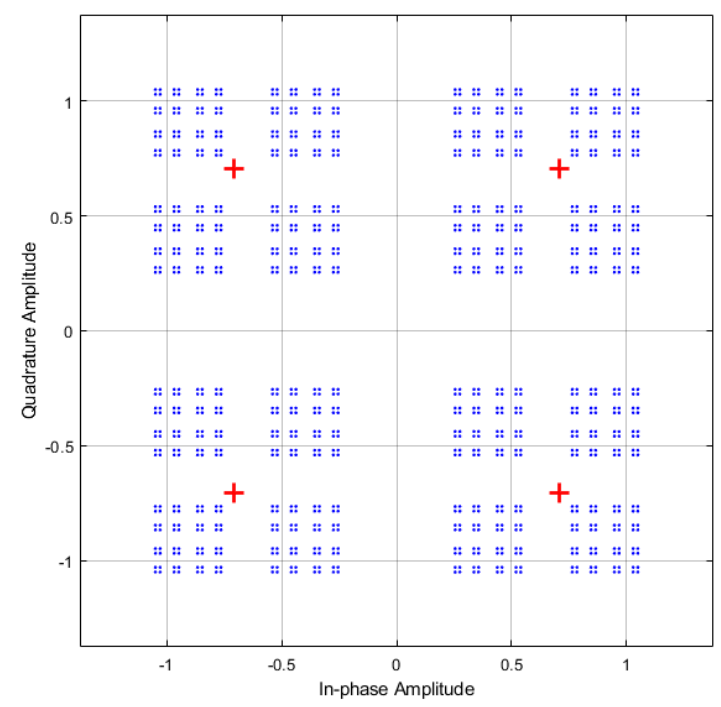

Fig. 8. 1024 superposition coded constellation points for 5 user multiplexing

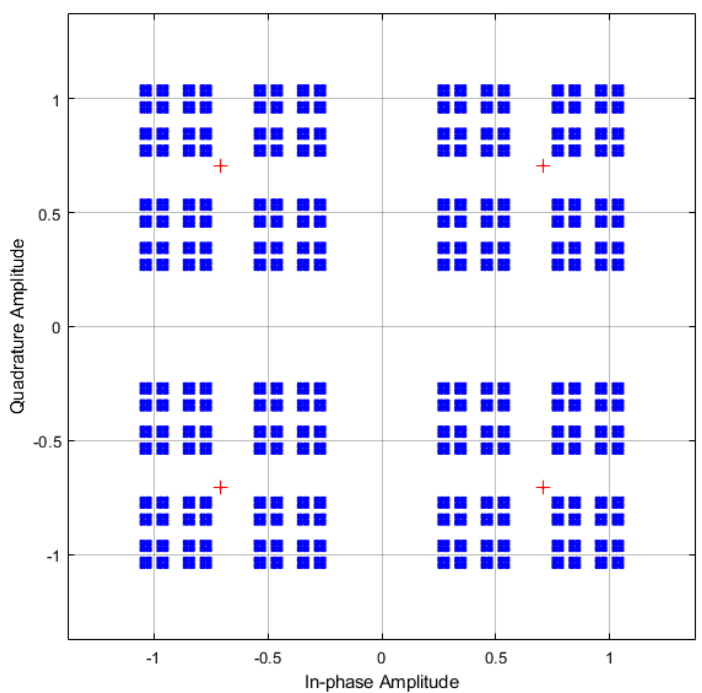

Fig. 9. 16384 superposition coded constellation points for 7 user multiplexing

The inphase and quadrature components of constellation points (symbols) are distorted as the superposition coded signal is transmitted through fading channels and exposed to random noise. Therefore, plotting each sample after equalization yields the distorted version of superposition coded signal. However, successive interference cancellation (SIC) 
helps to recover the original symbol scheme by removing the interference signals as we can see in Fig. 10 and Fig. 11 with reference QPSK 4 symbols plotted in the center. Different colors represent the constellation points conditioning at each successive iterations and the optimum recovery of SC constellation plane.

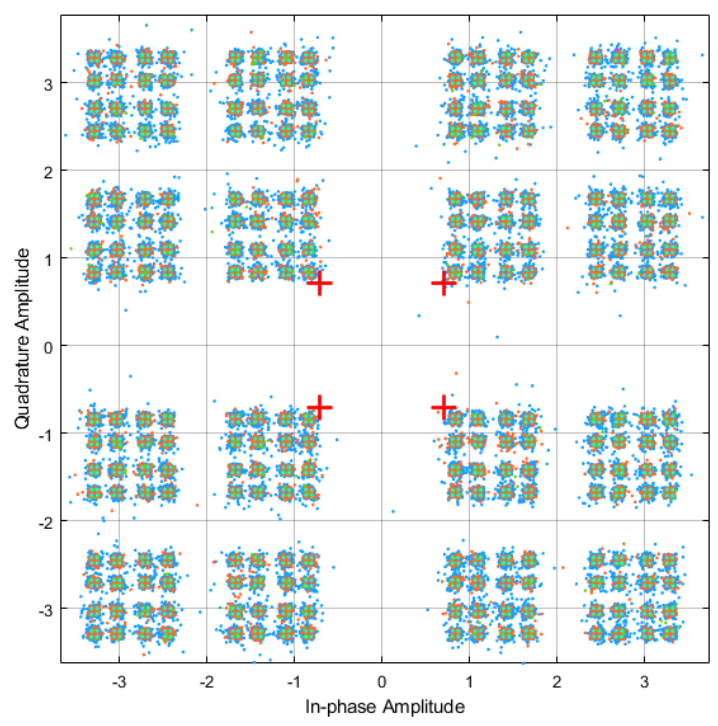

Fig. 10. Successive iterations of SIC for 5 user multiplexing - Green signal shows the recovered original SC mapping after complete SIC

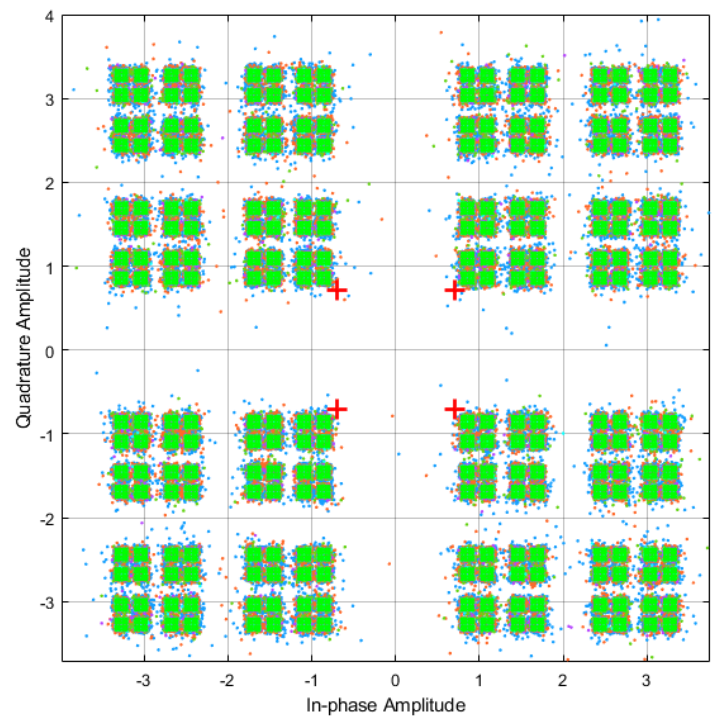

Fig. 11. Successive iterations of SIC for 7 user multiplexing - Green signal shows the recovered original SC mapping after complete SIC

\section{Closed-Form Expression for Bit Error Rate for Generic NOMA System}

Aiming to obtain a generalized expression for bit error rate of a generic NOMA communication system rendering services to $n$ - user, we examine the probabilistic nature of the received signal which is dependent upon two sequences of independent random variables, Rayleigh fading channel and AWGN noise channel. First we rephrase the equation (13) in terms of random variables for particular values for $k^{t h}$ user.

$$
\boldsymbol{y}_{\boldsymbol{k}}=\underbrace{\boldsymbol{H}_{\boldsymbol{k}} S_{k}}_{\text {Desired Signal }}+\underbrace{\boldsymbol{N}_{\boldsymbol{k}}}_{\text {Undesired Signal }}
$$

$\left.N_{\boldsymbol{k}}\right|_{n_{k}}$ is a random variable for noise distribution with particular value of $n_{k}$ for a certain symbol for $k^{\text {th }}$ user, correspondingly $\left.\boldsymbol{H}_{\boldsymbol{k}}\right|_{h_{k}}$ is a random variable for Rayleigh fading channel distribution with particular value $h_{k}$ as channel coefficient for the same symbol while $S_{k}$, the recovered signal for $k^{\text {th }}$ user after successful SIC is considered as a deterministic variable in operation defined in equation (19). If the undesired part (noise) exceeds the desirable part (product of channel and signal) of received signal then it is impossible to decipher the intended message, so erroneous bit is generated at user domain. The probability of erroneous bits in complete transmission session, bit error rate for $k^{\text {th }}$ user among the subgroup of $n$ users can be defined as:

$$
\begin{aligned}
\left.B E R\right|_{k} & =P\left(\boldsymbol{N}_{\boldsymbol{k}}>\boldsymbol{H}_{\boldsymbol{k}} S_{k}\right) \\
& =P\left(\frac{\boldsymbol{N}_{\boldsymbol{k}}}{\boldsymbol{H}_{\boldsymbol{k}}}>S_{k}\right)
\end{aligned}
$$

The recovered signal $S_{k}$ is obtained by successive removal of farther users (stronger ones) from the received signal $y_{k}$ as

$$
\begin{aligned}
S_{k} & =y_{k}-\sum_{j=1}^{k-1} \sqrt{\alpha_{j} P_{t}} s_{\text {mod }, j} \\
& =\sum_{j=k}^{n} \sqrt{\alpha_{j} P_{t}} s_{m o d, j}+n_{k} \bar{h}^{\dagger}
\end{aligned}
$$

The closed form expression of the probabilistic problem discussed in equation (20) is defined in Lemma 1.

Lemma I: For user $k$ located at radial distance $d_{k}$ from the base station with transmission power $P_{t}$, the bit error rate (BER) is given as

$$
\left.B E R\right|_{k}=\frac{1}{\pi} \sqrt{\frac{n_{0}}{d_{k}^{-\eta}}}\left(\frac{1}{2}-\frac{S_{k}\left(\pi-2 \tan ^{-1}\left(S_{k}\right)\right)}{4}\right)
$$

Proof: Please, see Appendix A

The analysis of equation (22) reveals dependence of BER $\left(\left.B E R\right|_{k}\right)$ of a user upon SIC recovered signal (equation (21)), transmission power $P_{t}$ base station and user radial distance $d_{k}$. Now we consider this analytical expression for proposed Fibonacci algorithm for $n$ user NOMA system and formulate relationship of BER with Fibonacci polynomial for particular SIC recovered signal $\left(S_{k}\right)$ as defined in Lemma 2.

Lemma II: For user $k$, SIC recovered signal $\left(S_{k}\right)$ is related to Fibonacci polynomial defined for $n$ users with order $N / 2$ as given below

$$
S_{k}=\frac{\beth_{n-(k-1)}^{N / 2}}{\beth_{n}^{N / 2}} \sqrt{P_{t}} \times s_{\text {mod }, j}+n_{k} \bar{h}^{\dagger}
$$

Proof: Please, see Appendix B.

The analytical BER results are presented in Fig. 12 for 7 user scenario with power coefficients assigned by Fibonacci 
allocation algorithms in equations $\sqrt{22}$ (23), alongwith simulation results for better understanding. The average BER analysis for 7 user NOMA system for both analytical and simulation cases are provided in Fig. 13, which shows the monotonic decrease in theoretical and practical dominions. As the transmission power increases $\left(P_{t} \rightarrow \infty\right)$, the simulation results converge to analytical characteristic curve. It is further observed that for equidistant users, the average Fibonacci algorithm simulation BER forms offset curve (parallel curve) with respect to the analytical curve as shown in Fig. 14 It represents the worst case with maximum difference from analytical curve because radius vector has zero variation for particular mean distance for deterministic allocation scheme, while all other distance choice will have certain variance. The more variance in radius vector leads to more rapid convergence to analytical curve. Considering these facts, well scattering of users of a subgroup in radial distance will conform to idealistic performance of Fibonacci allocation algorithm.

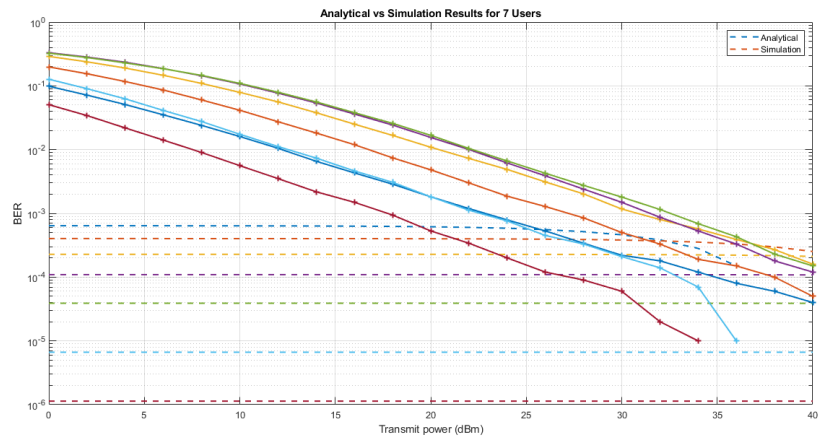

Fig. 12. Analytical and simulation BER results for individual 7 users

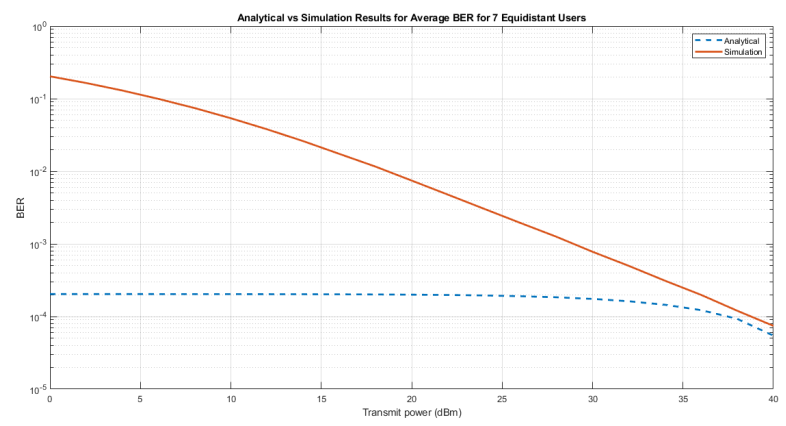

Fig. 13. Analytical and simulation average BER results for 7 users

\section{Comparison with CSI based Allocation}

For adjudging the effectiveness of the proposed Fibonacci power allocation algorithm, we compare its results with algorithms based on channel state information (CSI), which use channel parameters for assigning coefficients to each user at both superposition coding and successive interference cancellation stages. Two comparison criteria have been used to evaluate performance of these algorithms, bit error rate (BER)

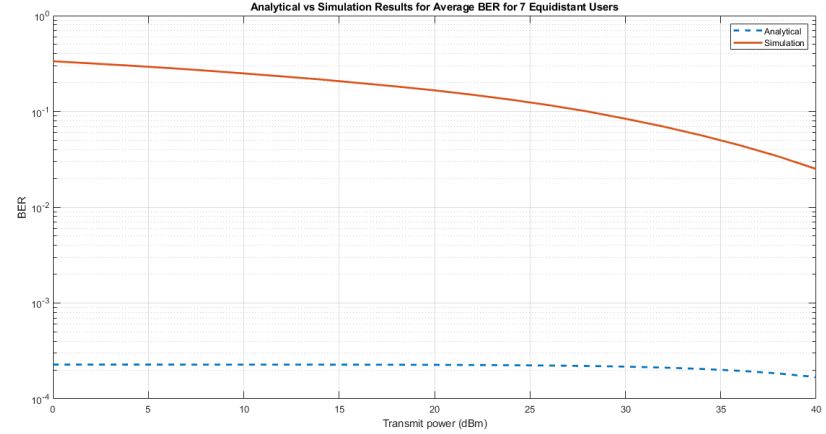

Fig. 14. Analytical and simulation average BER results for 7 equidistant users $d_{1}, d_{2}, \cdots, d_{n}=80$ meters in single arc

analysis for communication reliability and simulation time for analyzing the celerity of schemes in extreme real-time communication. Channel inversion is one the most rudimentary algorithms used for CSI based power allocation. It draws its basis from principle of fair power assignment as the power share for each user should be inversely related to the fading channel coefficients, so that users with adverse conditions are favoured with larger power proportion. The authors in [8] have devised a simplistic equation for allocation coefficients based on normalization of fading channel coefficients as

$$
\alpha_{k}=\frac{\frac{1}{\left|h_{k}\right|^{2}}}{\sum_{i=1}^{n} \frac{1}{\left|h_{i}\right|^{2}}}
$$

As we observe equation (24), it clearly points out to three important considerations. Firstly, significant amount of time will be consumed in estimation of channel coefficients and processing of this algorithm. Secondly, difference in estimation process at base station and users will lead to distinct sets of allocation coefficients, resulting in more erroneous data recovery by users while performing SIC. Finally, variation in positions of users will change the channel parameters, forcing into re-evaluation of power allocation coefficients.

The CSI based power allocation has been simulated and applied to the NOMA communication system with same specifications as mentioned in Table II maintaining the impartiality in comparative analysis. At this stage, we have only accounted for the first of three consideration discussed above. It has been assumed that base station and users have perfect knowledge of CSI and the positions of users are stationary for our analysis. BER curve for channel inversion scheme has been simulated in Fig. 15 for 7 users case NOMA for same user distances as followed in Fig. 7 It is noted that there was an increase in simulation time from 209 seconds for Fibonacci algorithm 7 user case to 231 seconds for channel inversion CSI 7 user NOMA, owing to fading channel vector computations involved in equation (24). The authors of this CSI algorithm in [8] have claimed BER values of order $10^{-4}$ for 3 user NOMA system which makes proposed Fibonacci allocation algorithm a favourable choice.

Visualization of average BER for 7 users for two algorithm is compared in Fig. 16 alongwith analytical BER curve, again 


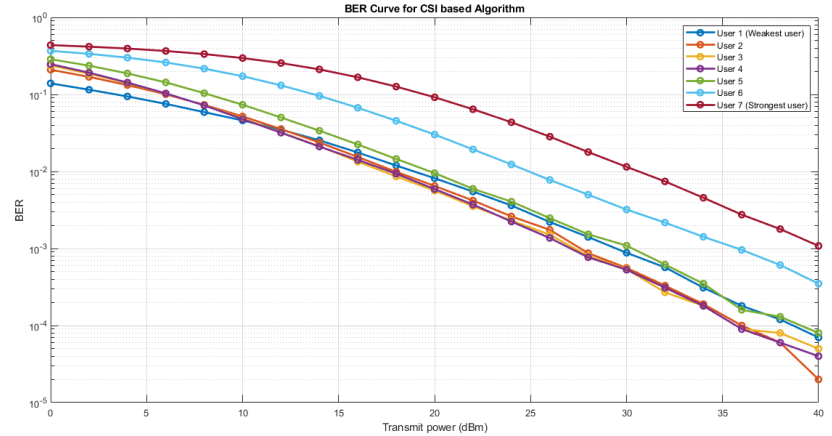

Fig. 15. BER results for CSI based algorithm for individual 7 users for allocation coefficients $(\alpha): 0.6373,0.2561,0.0839,0.0199,0.0026,8.19 \times$ $10^{-5}, 2.57 \times 10^{-6}$-Simulation time - $231 \mathrm{sec}$

iterating the exposition that Fibonacci allocation algorithm performance conforms to analytical BER valuation remarkably well in comparison to Channel inversion CSI algorithm.

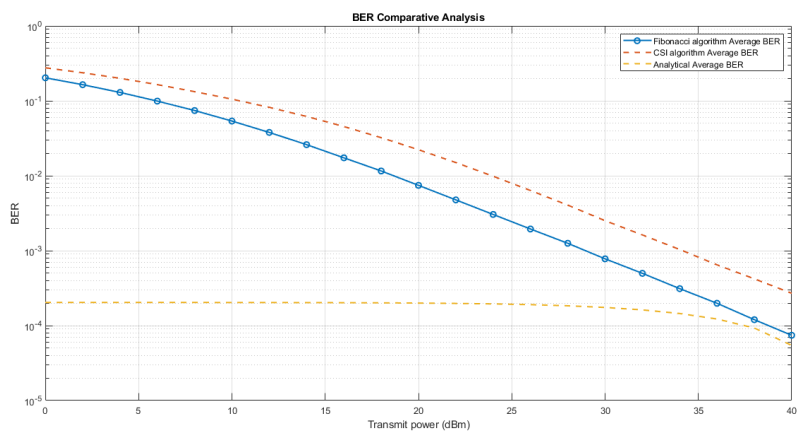

Fig. 16. Average BER comparative analysis of Fibonacci deterministic algorithm and Channel inversion CSI algorithm

\section{CONCLUSION}

In this research, a reliable and efficient power allocation algorithm for NOMA systems has been proposed which is based on a deterministic polynomial of Fibonacci number sequence. The proposed algorithm has been implemented to achieve power domain multiplexing on a simulated NOMA communication system for $n$ users in a single subgroup for which single transmission frequency is intended. BER curves have been analyzed for three scenarios (3,5 and 7 users/ subgroup) on this NOMA system with proposed coefficients. Numerical analysis for Bit error rate (BER) of generic NOMA system has been carried out to derive a closed form expression based on probabilistic models for Rayleigh fading channel and AWGN noise channel. Numerical and simulation results for proposed allocation algorithm have been investigated simultaneously and important corollaries have been deduced regarding the behaviour of the algorithm with respect to user scattering. Furthermore, channel inversion, a CSI based power allocation algorithm was compared with proposed scheme in terms of reliability (BER comparative analysis) and latency (simulation time comparison) which clearly pointed out the superior performance of Fibonacci allocation algorithm. In continuation for possible future endeavours, this algorithm can be employed for combination of power domain NOMA with MIMO techniques to achieve diversity in space domain.

\section{APPENDIX A \\ PROOF OF LEMMA I}

From equation 20, BER is analyzed which is the tail distribution of the ratio of two complex normal distributed random variable. Let the ratio of $\boldsymbol{N}_{\boldsymbol{k}} / \boldsymbol{H}_{\boldsymbol{k}}$ be equal to new complex random variable $Z$ such as: From equation 25,

$$
\begin{aligned}
\boldsymbol{Z} & =\frac{\boldsymbol{N}_{\boldsymbol{k}_{r}}+j \boldsymbol{N}_{\boldsymbol{k} i}}{\boldsymbol{H}_{\boldsymbol{k}_{r}}+j \boldsymbol{H}_{\boldsymbol{k}} i} \\
& =\boldsymbol{Z}_{\boldsymbol{r}}+j \boldsymbol{Z}_{\boldsymbol{i}}
\end{aligned}
$$

This complex vector will have probability density function (PDF) for zero mean $\boldsymbol{N}_{\boldsymbol{k}_{r}}, \boldsymbol{N}_{\boldsymbol{k} i}, \boldsymbol{H}_{\boldsymbol{k}_{r}}$ and $\boldsymbol{H}_{\boldsymbol{k}_{i}}$ as mentioned in [29] as following.

$$
f_{N / H}(z)=\frac{\sigma_{N_{k_{r}}}^{2}}{\pi \sigma_{H_{k r}}^{2}} \sqrt{\frac{n_{0}}{d_{k}^{-\eta}}}\left(|z|^{2}+\frac{\sigma_{N_{k i}}^{2}}{\sigma_{N_{k i}}^{2}}\right)^{-2}
$$

As $\sigma_{N_{k_{r}}}=\sigma_{H_{k_{r}}}$ and $\sigma_{N_{k_{r}}}=\sigma_{H_{k_{r}}}$, therefore equation 26 becomes,

$$
f_{N / H}(z)=\frac{1}{\pi} \sqrt{\frac{n_{0}}{d_{k}^{-\eta}}}\left(\frac{1}{\left(|z|^{2}+1\right)}\right)^{2}
$$

From equation 20, BER is Q-function of complex random vector $\boldsymbol{Z}$ as:

$$
\left.B E R\right|_{k}=P\left(\frac{\boldsymbol{N}_{\boldsymbol{k}}}{\boldsymbol{H}_{\boldsymbol{k}}}>S_{k}\right)=P\left(\boldsymbol{Z}>S_{k}\right)
$$

Substitution and integration of PDF of complex random vector $\boldsymbol{Z}$ in equation (28) gives

$$
\begin{aligned}
\left.B E R\right|_{k} & =P\left(\boldsymbol{Z}>S_{k}\right) \\
& =\frac{1}{\pi} \sqrt{\frac{n_{0}}{d_{k}^{-\eta}}} \int_{S_{k_{i}}}^{+\infty} \int_{S_{k_{r}}}^{+\infty} \frac{1}{\left(|z|^{2}+1\right)^{2}} d z_{r} d z_{i} \\
& =\frac{1}{\pi} \sqrt{\frac{n_{0}}{d_{k}^{-\eta}}}\left(\frac{1}{2}-\frac{S_{k}\left(\pi-2 \tan ^{-1}\left(S_{k}\right)\right)}{4}\right)
\end{aligned}
$$

Substituting $S_{k}$ from equation (21) in equation (31) will lead to BER for $k^{\text {th }}$ user showing its dependence upon transmission power and radial distance as:

$$
S_{k}=\sum_{j=k}^{n} \sqrt{\alpha_{j} P_{t}} s_{\text {mod }, j}+n_{k} \bar{h}^{\dagger}
$$

The proof is completed here.

\section{APPENDIX B}

\section{PROOF OF LEMMA II}

For deriving SIC recovered signal for Fibonacci power allocation algorithm, we use the SIC equation for generic NOMA system and move towards the particular solution. We substitute Fibonacci power allocation coefficients from equation (5) to (32) as

$$
S_{k}=\sum_{j=k}^{n} \sqrt{\frac{x_{n-(j-2)}^{N}}{\beth_{n}^{N}} P_{t}} \times s_{\text {mod }, j}+n_{k} \bar{h}^{\dagger}
$$


Consider summation term individually as

$$
\begin{aligned}
\sum_{j=k}^{n} \sqrt{\frac{x_{n-(j-2)}^{N}}{\beth_{n}^{N}}} & =\frac{x_{2}^{N / 2}+x_{3}^{N / 2}+\cdots+x_{n-(k-2)}^{N / 2}}{\beth_{n}^{N / 2}} \\
& =\frac{\beth_{n-(k-1)}^{N / 2}}{\beth_{n}^{N / 2}}
\end{aligned}
$$

Substituting equation (35) into (33) gives the requisite expres-

sion for SIC recovered signal for $k^{t h}$ user in terms of $N / 2$ order Fibonacci polynomial as

$$
S_{k}=\frac{\beth_{n-(k-1)}^{N / 2}}{\beth_{n}^{N / 2}} \sqrt{P_{t}} \times s_{\text {mod }, j}+n_{k} \bar{h}^{\dagger}
$$

which completes the proof.

\section{REFERENCES}

[1] "Proposed solutions for new radio access," Mobile and wireless communications enablers for the 2020 information society (METIS), Deliverable D.2.4, Feb. 2015.

[2] G. A. Akpakwu, B. J. Silva, G. P. Hancke, and A. M. Abu-Mahfouz, "A survey on $5 \mathrm{G}$ networks for the Internet of Things: Communication technologies and challenges," IEEE Access, vol. 6, pp. 3619-3647, 2018.

[3] Z. Ding and V. Poor, "Design of massive-MIMO-NOMA with limited feedback," IEEE Signal Process. Lett., vol. 23, no. 5, May 2016.

[4] Z. Ding, F. Adachi, and H. V. Poor, "The application of MIMO to nonorthogonal multiple access," IEEE Trans. Wireless Commun., vol. 15, no. 1, pp. 537-552, Jan. 2016.

[5] A. Sousa de Sena, D. B. da Costa, Z. Ding, P. H. J. Nardelli, U. S. Dias and C. B. Papadias, "Massive MIMO-NOMA Networks With Successive Sub-Array Activation," in IEEE Transactions on Wireless Communications, vol. 19, no. 3, pp. 1622-1635, March 2020.

[6] "Study on Non-Orthogonal Multiple Access (NOMA) for NR" (Release 16), 3GPP, Specification: 38.812, V16.0.0. 2018.

[7] Q. He, Y. Hu, and A. Schmeink, "Closed-form symbol error rate expressions for non-orthogonal multiple access systems," IEEE Trans. Veh. Technol., vol. 68, no. 7, pp. 6775 - 6789, Jul. 2019.

[8] M. M. El-Sayed, A. S. Ibrahim and M. M. Khairy, "Power allocation strategies for Non-Orthogonal Multiple Access," 2016 International Conference on Selected Topics in Mobile and Wireless Networking (MoWNeT), Cairo, 2016.

[9] Z. Yang, Z. Ding, P. Fan and G. K. Karagiannidis, "On the Performance of Non-orthogonal Multiple Access Systems With Partial Channel Information," in IEEE Transactions on Communications, vol. 64, no. 2, pp. 654-667, Feb. 2016.

[10] U. Ozmat, O. Ulgen and E. Gunaydin, "Bit Error Rate Analysis of NonOrthogonal Multiple Access (NOMA) Technique in 5G with Different Power and User Scenarios," 2018 Advances in Wireless and Optical Communications (RTUWO), Riga, 2018.

[11] Z. Tang, L. Sun, L. Cao, S. Qi and Y. Feng, "Reconsidering Design of Multi-Antenna NOMA Systems With Limited Feedback," in IEEE Transactions on Wireless Communications, vol. 19, no. 3, pp. 1519-1534, March 2020.

[12] T. Manglayev, R. C. Kizilirmak and Y. H. Kho, "Optimum power allocation for non-orthogonal multiple access (NOMA)," 2016 IEEE 10th International Conference on Application of Information and Communication Technologies (AICT), Baku, 2016.

[13] N. Yang, H. Zhang, K. Long, H. Hsieh and J. Liu, "Deep Neural Network for Resource Management in NOMA Networks," in IEEE Transactions on Vehicular Technology, vol. 69, no. 1, pp. 876-886, Jan. 2020.

[14] Y. Zhang, X. Wang and Y. Xu, "Energy-Efficient Resource Allocation in Uplink NOMA Systems with Deep Reinforcement Learning," 2019 11th International Conference on Wireless Communications and Signal Processing (WCSP), Xi'an, China, 2019.

[15] Yi Ma, Na Yi and R. Tafazolli, "Channel estimation for PRP-OFDM in slowly time-varying channel: first-order or second-order statistics?", in IEEE Signal Processing Letters, vol. 13, no. 3, pp. 129-132, March 2006.

[16] H. A. David and H. N. Nagaraja, Order Statistics, 3rd ed. Wiley Series in Probability and Statistics, Aug. 2003.
[17] B. Sklar, "Rayleigh fading channels in mobile digital communication systems .I. Characterization," in IEEE Communications Magazine, vol. 35, no. 7, pp. 90-100, July 1997.

[18] E. Matricciani, "A Model of the Probability Distribution of the Signal-toNoise Ratio Estimated from BER Measurements," 2011 IEEE Vehicular Technology Conference (VTC Fall), San Francisco, CA, 2011.

[19] Z. Q. Al-Abbasi and D. K. C. So, "Power allocation for sum rate maximization in non-orthogonal multiple access system," 2015 IEEE 26th Annual International Symposium on Personal, Indoor, and Mobile Radio Communications (PIMRC), Hong Kong, 2015.

[20] P. Kyritsi, P. Stoica, G. Papanicolaou, P. Eggers and A. Oprea, "Time Reversal and Zero-Forcing Equalization for Fixed Wireless Access Channels," Conference Record of the Thirty-Ninth Asilomar Conference on Signals, Systems and Computers, 2005.

[21] C. B. Ribeiro, M. L. R. de Campos and P. S. R. Diniz, "Zero-forcing equalization for time-varying systems with memory," 2004 IEEE International Symposium on Circuits and Systems (IEEE Cat. No.04CH37512), Vancouver, BC, 2004.

[22] E. Attang, Y. Wu and G. E. Atkin, "Signal sets for constellation expansion in NOMA," 2017 IEEE International Conference on Electro Information Technology (EIT), Lincoln, NE, 2017.

[23] L. Jiang, X. Li, N. Ye and A. Wang, "Deep Learning-Aided Constellation Design for Downlink NOMA," 2019 15th International Wireless Communications and Mobile Computing Conference (IWCMC), Tangier, Morocco, 2019.

[24] J. Zhang, X. Wang, T. Hasegawa and T. Kubo, "Downlink NonOrthogonal Multiple Access (NOMA) Constellation Rotation," 2016 IEEE 84th Vehicular Technology Conference (VTC-Fall), Montreal, QC, 2016.

[25] T. Assaf, A. Al-Dweik, M. E. Moursi and H. Zeineldin, "Exact BER Performance Analysis for Downlink NOMA Systems Over Nakagami- $m$ Fading Channels," in IEEE Access, vol. 7, pp. 134539-134555, 2019.

[26] F. Yilmaz, "On the Relationships Between Average Channel Capacity, Average Bit Error Rate, Outage Probability, and Outage Capacity Over Additive White Gaussian Noise Channels," in IEEE Transactions on Communications, vol. 68, no. 5, pp. 2763-2776, May 2020.

[27] S. Ali, E. Hossain and D. I. Kim, "Non-Orthogonal Multiple Access (NOMA) for Downlink Multiuser MIMO Systems: User Clustering, Beamforming, and Power Allocation," in IEEE Access, vol. 5, pp. 565577, 2017.

[28] B. Di, L. Song and Y. Li, "Sub-Channel Assignment, Power Allocation, and User Scheduling for Non-Orthogonal Multiple Access Networks," in IEEE Transactions on Wireless Communications, vol. 15, no. 11, pp. 7686-7698, Nov. 2016.

[29] R. J. Baxley, B. T. Walkenhorst and G. Acosta-Marum, "Complex Gaussian Ratio Distribution with Applications for Error Rate Calculation in Fading Channels with Imperfect CSI," 2010 IEEE Global Telecommunications Conference GLOBECOM 2010, Miami, FL, 2010. 